\title{
纳米多孔金的单分子层门控离子输运性质研究
}

\author{
间娜娜肖天亮刘兆阅* \\ (北京航空航天大学化学学院 北京 100191)
}

\begin{abstract}
摘要 生物离子通道能够对环境刺激作出响应, 有效地调节细胞内外的物质平衡, 保证体内的正常生命活动. 研究具 有生物离子通道功能的人工离子通道对发展离子开关具有重要的意义. 本工作利用电化学去合金法制备了具有三维通 道结构的纳米多孔金膜，并将其应用于离子通道，研究其离子输运性质. 在电场作用下，纳米多孔金由于发生极化而 使通道表现出离子整流性质. 在纳米多孔金表面修饰十二烷基硫醇单分子层, 利用其疏水效应, 通道能够阻止离子的 传输, 使其处于 “关闭” 态. 溶液中的化学刺激如表面活性剂能够增强电解质溶液在通道表面的浸润, 有利于离子的传 输，使通道处于 “打开” 态. 这种单分子层修饰的纳米多孔金表现出表面活性剂响应的离子开关特性.
\end{abstract}

关键词 离子通道; 去合金法; 纳米多孔金; 表面活性剂; 离子开关

\section{Monolayer-gated Ion Transport in Artificial Ion Channels Based on A Nanoporous Gold Membrane}

\author{
Yan, Nana Xiao, Tianliang Liu, Zhaoyue* \\ (School of Chemistry, Beihang University, Beijing 100191)
}

\begin{abstract}
Biological ion channels that intelligently control the transport of ions or molecules through the cell membrane in response to external stimuli can maintain the balance between the extracellular and intracellular substances, which ensures the normal life activities of the organism. The development of artificial ion channels with analogous function to the biological counterparts is of great significance because of their possible applications as ion switches and sensors. In this paper, we describe a new type of artificial ion channels based on de-alloyed nanoporous gold membrane with three-dimensional nanochannels. The nanochannels were built by electrochemical etching of gold-silver alloy in concentrated nitric acid. The surface morphology and component of nanoporous gold membrane were characterized by scanning electron microscope (SEM) and energy dispersive X-ray spectrum (EDX). The ion transport properties of artificial ion channels were characterized with current-voltage curves which was measured by a picoammeter. Our results indicate that this nanoporous gold membrane demonstrates an ion rectification phenomenon because of the electrochemical polarization of gold under an electric field. Subsequently, a layer of hydrophobic molecules was assembled on the surface of nanoporous gold membrane by strong $\mathrm{Au}$-thiol bonds after immersed in the solution of 1-dodecanethiol. The measurements of water contact angles (CAs) indicated that the modification of 1-dodecanethiol molecules converted the surface water CA of nanoporous gold membrane from $36.5^{\circ}$ to $120.6^{\circ}$. This hydrophobic monolayer prevents the transport of water-soluble ions, which makes the channels exist in an "off" state. The stimulus of surfactant in the electrolyte is favorable for the wetting of channel surface by aqueous electrolyte, which makes the channels exist in an "on" state for water-soluble ions. Therefore, the monolayer-modified nanoporous gold membrane can serve as a surfactant-gated ion switch. Our work provides a new idea for the preparation of artificial ion channels, which can be applied for intelligently responsive artificial system.

Keywords ion channel; de-alloying; nanoporous gold; surfactant; ion switch
\end{abstract}

\section{1 引言}

生物细胞膜内存在着功能各异的离子通道. 离子通 道是由膜蛋白分子和磷脂双分子层组装而成，能够智能 响应外界环境变化并调节膜内外离子的输运 ${ }^{[1 \sim 3]}$. 离子 的门控性、选择性和整流性是生物离子通道的三个重要 特性 ${ }^{[47]}$. 然而, 生物离子通道只能在特定生物体内环 境中发挥作用, 其结构的不稳定性限制了它在非生物环
境的应用. 受生物离子通道结构和原理的启发, 人们制 备出具有生物离子通道功能的多种人工纳米通道体 系 ${ }^{[8-21]}$. 这些人工纳米通道具有机械强度高、化学性质

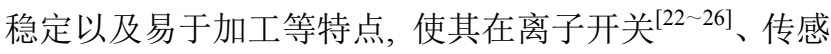

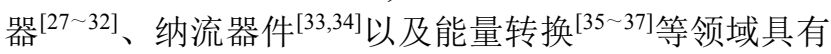
巨大的应用价值.

基于金材料的人工纳米通道以其优异的表面可修 饰性受到人们的广泛关注 ${ }^{[38 ~ 45]}$. Martin 等 ${ }^{[38]}$ 以重粒子轰

* E-mail: liuzy@buaa.edu.cn; Te1.: 010-82317801

Received May 17, 2017; published August 7, 2017.

Project supported by the National Natural Science Foundation of China (No. 21571011) and the National Basic Research Program of China (No. 2014CB931803).

项目受国家自然科学基金(No. 21571011)和国家重点基础研究发展计划(No. 2014CB931803)资助. 
击得到的聚碳酸酯柱形纳米孔为模板, 利用无电沉积的 方法制备了金纳米通道. 由于金对氯离子的选择性吸 附, 金纳米通道在氯化钾电解质中表现出阳离子选择 性. 进一步，他们在金纳米通道表面通过金颈基相互作 用修饰半胱氨酸, 实现了 $\mathrm{pH}$ 值门控的离子输运性质. Siwy 等 ${ }^{[39]}$ 通过在雉形的聚对苯二甲酸乙二醇酯(PET)单 纳米孔表面沉积金, 得到雉形的金纳米通道. 通过在其 表面修饰氨基, 实现了 $\mathrm{pH}$ 值门控的离子单向导通, 即 离子整流性. $\mathrm{Hou}$ 等 ${ }^{[40]}$ 在雉形 PET 单纳米通道的小孔端 沉积纳米颗粒来调控纳米孔的大小, 增强了纳米通道的 离子整流. 最近, Yang 和 Siwy 等 ${ }^{[44]}$ 报道了一种 $\mathrm{Au}-\mathrm{SiN}$ 纳米通道. 他们首先在 $\mathrm{SiN}$ 薄膜上沉积一层金, 然后采 用透射电子显微镜(TEM)下电子束刻蚀将其击穿, 形成 单纳米通道. 在电场作用下, 纳米通道中 $\mathrm{Au}$ 表面发生 极化现象而带有电荷, 从而使纳米通道表现出离子整流 现象. 然而, 目前金纳米通道的制备过程都包括复杂与 昂贵的微纳米加工技术, 如重离子轰击加径迹刻 蚀 ${ }^{[38 ~ 40]}$ 和 TEM 电子束刻蚀 ${ }^{[44]}$ 等. 因此, 有必要发展一 种简单易得的新型金纳米通道.

利用去合金法制备的纳米多孔金膜由于其内部具 有三维纳米通道结构而受到广泛关注 ${ }^{[46 ~ 50]}$. 纳米通道 的形成是通过对金银合金片中的银进行选择性刻蚀而 实现的. 在硝酸作用下, 化学性质较活泼的银以离子形 式进入电解质, 而化学性质较稳定的金自组装形成相互 交错的多孔网状结构 ${ }^{[48]}$. 去合金法避免了金纳米通道 必须附着在基底的缺点，可以用来制备自支持的纳米多 孔金膜 ${ }^{[46,49]}$. 这种多孔金膜已在催化和分子释放等领域 得到广泛的研究. 例如, $\mathrm{Xu}$ 等 ${ }^{[47]}$ 以纳米多孔金膜中的纳 米通道作为一氧化碳气体的传输通道, 金作为催化剂, 实现了一氧化碳在低温下的催化氧化. Polat 等 ${ }^{[50]}$ 以纳米 多孔金膜为荧光分子的吸附载体, 利用金和氯离子之间 较强的化学键合作用, 实现了氯离子门控的苂光分子释 放. 去合金法制备纳米多孔金膜的方法虽然已经比较成 熟, 但是其还没有被应用于人工离子通道来对离子的输 运进行调控.

本论文利用电化学去合金法制备了自支持的纳米 多孔金膜, 将其应用于人工离子通道, 研究了其离子输 运性质. 在电场作用下, 纳米多孔金表面由于发生极化 现象而使通道表现出离子整流性质. 金纳米通道中的离 子输运性质可以通过在其表面化学修饰十二烷基硫醇 单分子层来调控. 单分子层的疏水效应能够阻止离子的 传输, 使通道处于 “关闭” 态. 溶液中的表面活性剂能 够增强电解质溶液在疏水通道表面的浸润, 有利于离子 的传输, 使通道处于 “打开” 态.

\section{2 结果与讨论}

\section{1 纳米多孔金膜的结构表征}

采用如图 $1 \mathrm{~A}$ 所示的实验装置进行电化学去合金实
验. 其中，阳极为金银合金片，阴极为铂电极，电解质 为 $30 \%$ 的硝酸水溶液. 去合金反应在 $1 \mathrm{~V}$ 电压下进行 15 min. 在硝酸作用下, 化学性质较活泼的银以离子形式 进入电解质溶液中, 而留下化学性质较稳定的金. 图 $1 \mathrm{~B}$ 显示去合金前后金属片的颜色有明显的差别. 去合金之 前，金银合金片为银色. 银溶解后，得到的纳米多孔金 显示出均匀的暗红色，即金的颜色. 从反应后样品的表 面及截面(图 1C, 1D)的 SEM 照片观察可以发现, 纳米多 孔金呈现三维贯通多孔海绵状结构, 内部存在大量的纳 米通道，通道孔径大约在 $28 \sim 32 \mathrm{~nm}$ 左右. 纳米多孔金 膜的厚度约为 $35 \mu \mathrm{m}$. 我们发现去合金反应的时间对金 膜表面纳米孔的孔径影响不大. 去合金过程是一个自发 进行的不平衡过程，且可以发生于合金的任一部位. 随 着活泼的银元素进入溶液，剩下的金 “框架” 则形成连 续的多孔结构 ${ }^{[51]}$. 由于银在浓硝酸中的溶解速度较快, 延长去合金反应时间主要增加的是刻蚀深度，故对表面 纳米孔的孔径影响不大. 从图 $1 \mathrm{E}$ 的能谱图可以看出, 去合金前后谱线差别最大的是银元素，反应后银元素的 特征峰基本消失，说明合金中的大部分银原子已被溶 解 ${ }^{[52]}$. 最后得到的纳米多孔金表面呈现出亲水性, 水接 触角为 $36.5^{\circ}$ (图 $1 \mathrm{~F}$ ), 这样的亲水表面有利于水溶性离 子的传输.

\section{2 纳米多孔金膜的离子输运性质}

我们利用图 2A 所示装置来验证纳米多孔金膜中通 道的通透性. 图 $2 \mathrm{~A}$ 中左侧的池子里加入 $0.5 \mathrm{mmol} / \mathrm{L}$ 的 亚甲基蓝水溶液，右侧池子里加入超纯水，中间夹入纳 米多孔金膜. 将上述装置静置 $24 \mathrm{~h}$, 观察右侧超纯水颜 色的变化. 作为对比, 我们用金银合金片进行同样的渗 透实验. 如图 2B 所示, 经过 $24 \mathrm{~h}$ 渗透, 纳米多孔金膜可 以允许亚甲基蓝分子通过而使右侧超纯水变为蓝绿色. 而金银合金片完全阻止亚甲基蓝分子的透过，使超纯水 保持无色. 我们的结果证明了纳米多孔金内部的三维通 道是通透的.

本实验采用测试纳米多孔金膜的离子电流-电压 $(I \sim V)$ 曲线来表征其离子输运性能. 实验装置如图 $2 \mathrm{C}$ 所 示, 将纳米多孔金合金膜夹在两个电化学池中间, 通过 两个 $\mathrm{Ag} / \mathrm{AgCl}$ 电极对膜施加电压, 电解质溶液为 1 $\mathrm{mmol} / \mathrm{L}$ 的 $\mathrm{KF}$ 水溶液, 电压测试范围为 $-2 \mathrm{~V}$ 到 $+2 \mathrm{~V}$. 如图 2D 所示, 合金片表现出非线性的 $I \sim V$ 曲线. 由于 合金片内部并不存在可供离子传输的纳米通道, 因此我 们认为这种 $I \sim V$ 曲线的非线性是由金的极化效应引起 的. 在电场作用下(负向扫描)，钾离子聚集在阳极一侧 的金表面. 相反，氯离子聚集在阴极一侧的金表面. 钾 离子和氯离子在合金片两侧的聚集产生了一个内电场, 内电场将会破坏合金片中的电荷平衡，使合金片中的电 子沿着内电场的反方向移动. 这种极化过程在外电路产 生极化电流. 当电压扫描方向反转时(正向扫描), 负向 扫描时所产生的内电场将会抵消一部分外加电场，减少 

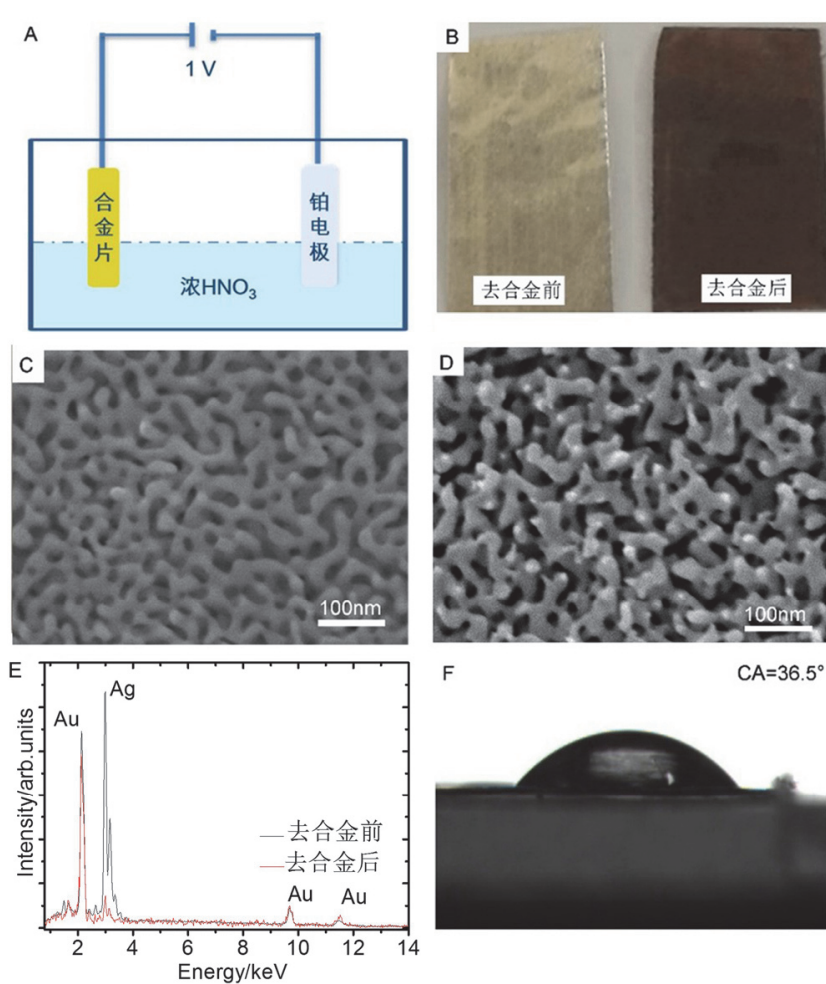

$\mathrm{F}$

$\mathrm{CA}=36.5^{\circ}$

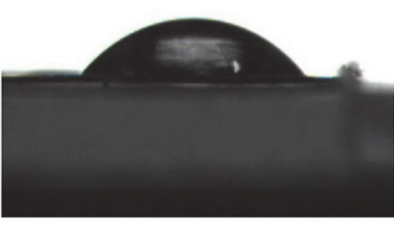

图 1 (A) 去合金装置图; (B) 金银合金片去合金前后的照片; (C) 纳 米多孔金表面扫描电镜图; (D) 纳米多孔金横截面扫描电镜图; (E) 金 银合金片去合金前后的能量色散 X 射线衍射图(EDX); (F) 纳米多孔 金的接触角

Figure 1 (A) Schematic setup for the de-alloying process; (B) Photograph of Au-Ag alloy sheet before and after de-alloying; (C) Top-view and (D) cross-sectional SEM image of the nanoporous gold; (E) Energy dispersive X-ray diffraction patterns of Au-Ag alloy sheet before and after de-alloying; (F) Contact angle (CA) of the nanoporous $\mathrm{Au}$

金的极化，从而产生了一个较小的极化电流. 合金片在 $-2 \mathrm{~V}$ 和 $+2 \mathrm{~V}$ 下电流绝对值之比为 1.40. 去合金反应 后, 纳米多孔金膜的电流有显著增加. 流过的电流主要 包括两部分, 即上述的极化电流和离子在纳米通道中传 输所引起的离子电流. 电流的增加是由离子电流引起 的. 在 $0 \mathrm{~V}$ 外压时, 纳米多孔金产生了正向电流(即, 零 点电流). 当流过纳米多孔金的电流为 0 时, 外加电压是 $-0.75 \mathrm{~V}$ (即, 零点电压). 在 $-2 \mathrm{~V}$ 和 $+2 \mathrm{~V}$ 下, 电流绝对 值之比为 1.88. 而且, 去合金反应的时间对 $I \sim V$ 曲线没 有明显的影响, 这主要是由于纳米多孔金的孔径随着反 应时间的变化较小. 电流非对称性的增加是由纳米通道 对离子输运的调控引起的. 当电压的极性由负切换到正 时, 负电压极化所产生的内电场能够抑制正电压下离子 在通道中的传输(图 3), 从而使 $I \sim V$ 曲线显示出更大的 非对称性. 和合金片相比, 纳米多孔金的零点电流有了 显著的提高. 这是由于纳米多孔金内部的大量纳米通道 表面被极化而聚集了更多的钾离子和氯离子. 在 $0 \mathrm{~V}$ 外 压时, 纳米通道表面去极化而产生一个和极化电压方向 相反的极化电流.
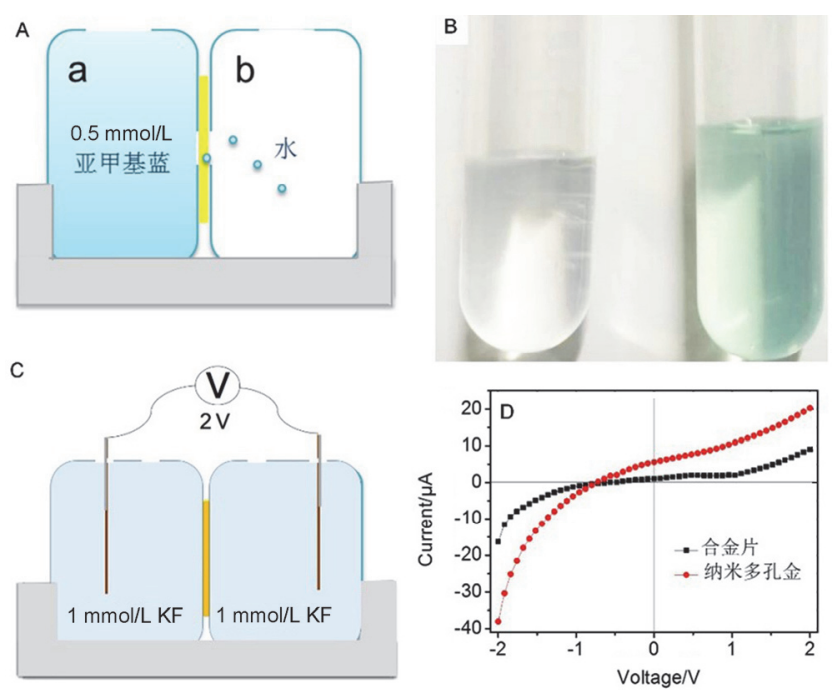

图 2 (A) 利用亚甲基蓝渗透实验来验证纳米多孔金通透性的装置示 意图; (B) 合金片和纳米多孔金膜渗透 $24 \mathrm{~h}$ 后, 图 A 中高纯水的颜色 变化照片. 纳米多孔金膜可以允许亚甲基蓝分子通过而使超纯水变为 蓝绿色. 而金银合金片完全阻止亚甲基蓝分子的透过，使超纯水保持 无色; (C) $I-V$ 曲线测试装置图; (D) 纳米多孔金及金银合金片的 $I-V$ 曲 线

Figure 2 (A) Schematic setup for the permeation of methylene blue; (B) After permeation of $24 \mathrm{~h}$, the color change of water in A; (C) Schematic setup for the measurement of $I-V$ curves; (D) $I-V$ curves of the nanoporous gold membrane and $\mathrm{Au}-\mathrm{Ag}$ alloy sheet
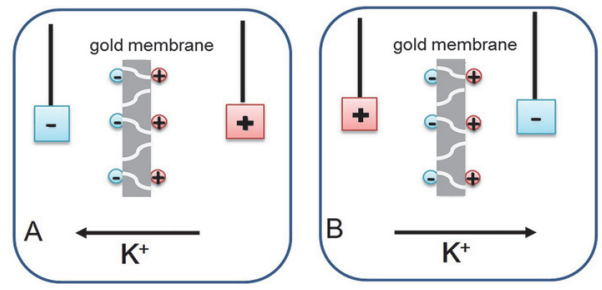

图 3 纳米多孔金极化整流的机理示意图. (A) 负电压扫描时, 正负 离子聚集在纳米多孔金两侧产生一个内电场; (B) 当电压的极性由负 切换到正时, 负电压极化所产生的内电场抑制了正电压下离子在通道 中的传输

Figure 3 Schematic mechanism for the polarization-induced ion rectification in nanoporous Au membrane. (A) When nanoporous Au membrane is subjected to a negative voltage, an internal electric field is formed across the membrane; (B) When the membrane is subjected to a positive voltage, the internal electric field will prevent the ion transport through the membrane

\section{3 表面活性剂调控的离子电流}

金纳米通道的一个重要优点是其优异的表面可修 饰性. 因此我们可以利用自组装的方法在纳米通道表面 修饰单分子层，从而调控通道的离子输运性质. 本实验 中，我们利用金和颈基之间的配位相互作用，在纳米多 孔金膜表面修饰十二烷基硫醇单分子层, 实现其对离子 输运的调控. 如图 4A 所示, 十二烷基硫醇分子修饰后, 金纳米通道的表面由亲水 $\left(36.5^{\circ}\right)$ 转变为疏水 $\left(120.6^{\circ}\right)$. 疏水的表面能够阻止离子在纳米通道中的传输, 使纳米 
通道产生一个较低的电流(图 4B), 这说明单层分子修饰 能够使金纳米通道对水溶性离子处于 “关闭” 状态.

我们知道, 水中表面活性剂能够有效降低水的表面 张力, 从而改进电解质溶液对纳米通道表面的浸润, 增 加水溶性离子在通道中的传输. 基于这一原理, 我们在 电解质中添加十二烷基苯磺酸钠表面活性剂(SDBS)使 纳米通道处于 “打开” 状态. 如图 4A 所示, 当电解质中 溶液中 SDBS 浓度增大到一定程度时 $\left(10^{-5} \mathrm{~mol} / \mathrm{L}\right)$, 电解 质在纳米通道表面的接触角变小, 对应所产生的离子电 流变大. 作为对比, 电解质中表面活性剂分子对没有疏 水分子修饰的纳米多孔金膜的离子电流影响较小. 我们 以 $-2 \mathrm{~V}$ 电压下的电流值计算了纳米通道的电阻值 $\left(R_{\mathrm{m}}\right)$, 见图 4C. 结果表明, 当浓度较低时, SDBS 的加入对纳 米通道的电阻基本没有影响, 说明低浓度的 SDBS 没有 明显改变电解质在通道表面的浸润. 当 SDBS 的浓度增 加到 $10^{-6} \mathrm{~mol} / \mathrm{L}$ 时, 可以发现电阻随着 SDBS 浓度的增 大迅速变小. 这主要是因为电解质中的表面活性剂有利 于电解质在疏水表面的浸润, 含有表面活性剂的电解质 在疏水纳米多孔金膜表面的接触角变小 (图 4A), 使电解 质溶液更容易通过纳米孔道, 故而电流增大, 等效电阻 变小. 单分子层修饰后的纳米多孔金作为人工离子通道 具有表面活性剂响应的特性.
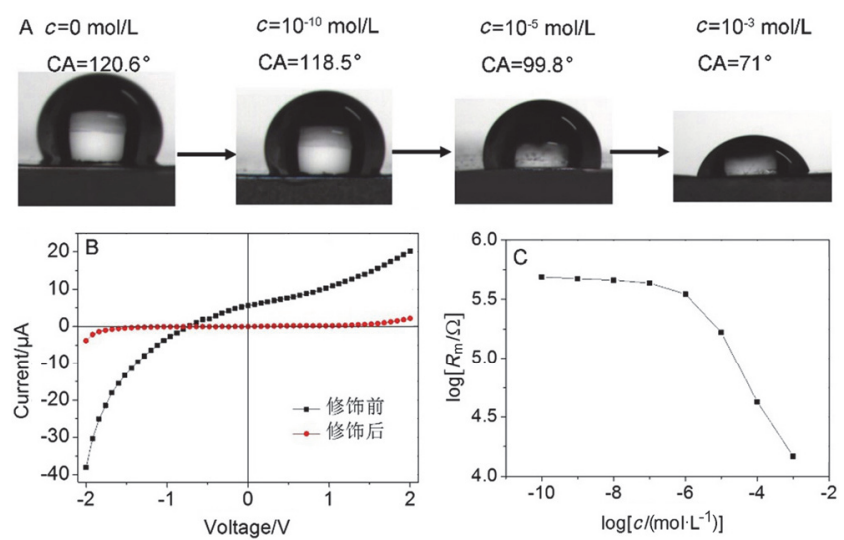

图 4 (A) 含有不同浓度的十二烷基苯磺酸钠(SDBS)电解质在十二烷 基硫醇修饰的纳米多孔金膜表面的接触角, SDBS 的浓度分别为 0 , $10^{-10}, 10^{-5}$ 和 $10^{-3} \mathrm{~mol} / \mathrm{L}$. (B) 纳米多孔金膜在十二烷基硫醇修饰前后 的 $I-V$ 曲线. (C) 在不同 SDBS 浓度下, 以 $-2 \mathrm{~V}$ 电压下的电流值计算 的十二烷基硫醇修饰的纳米多孔金膜的电阻值 $\left(R_{\mathrm{m}}\right)$

Figure 4 (A) The water contact angles of KF electrolyte with different concentrations $(c)$ of dodecylbenzenesulphonate sodium (SDBS) on the surface of 1-dodecanethiol-modified nanoporous $\mathrm{Au}$ membrane (thiolAu). $c=0,10^{-10}, 10^{-5}$ and $10^{-3} \mathrm{~mol} / \mathrm{L}$. (B) The $I-V$ curves of Au membrane in $\mathrm{KF}$ electrolyte before and after thiolation. (C) The equivalent resistance $\left(R_{\mathrm{m}}\right)$ of thiol-Au membrane in KF electrolyte with different concentrations of SDBS calculated by the current value at a voltage of $-2 \mathrm{~V}$

\section{3 结论}

本论文利用去合金法制备了纳米多孔金膜，并将其 应用于人工离子通道中. 结果表明, 纳米多孔金膜在电
场作用下能够发生极化. 极化作用所产生的内电场能够 使多孔膜表现出整流效应. 纳米多孔金表面的十二烷基 硫醇单分子层使通道呈现疏水性，阻碍离子的传输，使 通道呈现 “关闭” 的状态. 电解质中的表面活性剂能够 改进电解质溶液对纳米通道表面的浸润, 使通道处于 “打开”状态，导致流过通道的电流增大，等效电阻变小. 这种单分子层修饰的纳米多孔金膜表现出表面活性剂 响应的开关性质.

\section{4 实验部分}

\section{1 纳米多孔金膜的制备与表征}

本实验使用 $\mathrm{Au}$ 和 $\mathrm{Ag}$ 的质量百分含量分别为 $42 \%$ 和 $58 \%$ 的金银合金片(常熟市常宏贵金属有限公司)来制 备纳米多孔金. 为减少合金的内部应力 ${ }^{[47]}$, 去合金化反 应之前将金银合金片在 $850{ }^{\circ} \mathrm{C}$ 下焙烧 $10 \mathrm{~h}$ ，以使金和银 原子在合金内部扩散均匀. 然后使用超纯水和丙酮溶液 超声清洗金银合金片，干燥后备用. 以金银合金片作为 阳极，铂片作为阴极，硝酸溶液(质量百分含量 $30 \%$ ) 为 电解质，在 $1 \mathrm{~V}$ 电压下进行去合金反应，反应时间控制 为 $15 \mathrm{~min}$. 通过 JEOL JSM-7500F 场发射扫描电子显微 镜(SEM)观察纳米多孔金纳米的微观形貌, 采用能量色 散 X 射线衍射图(EDX)对其表面元素进行分析.

\section{2 纳米多孔金膜的通透性实验}

我们通过观察亚甲基蓝染料在纳米多孔金膜中的 渗透来验证膜的通透性. 将金膜固定在两个电化学池中 间，其中一个池子中注入 $0.5 \mathrm{mmol} / \mathrm{L}$ 的亚甲基蓝水溶 液, 另一个池子中注入超纯水. 将上述电化学池静置 24 $\mathrm{h}$, 观察超纯水一侧的颜色变化. 作为对比, 将未去合金 的金银合金片进行相同的通透性实验，观察超纯水的颜 色变化.

\section{3 离子输运及门控性质}

纳米多孔金膜的离子输运性质通过测量其离子电 流-电压 $(I \sim V)$ 曲线来表征. 将纳米多孔金膜固定在两 个电化学池中间, 电化学池中注入 $1 \mathrm{mmol} / \mathrm{L}$ 的 KF 电解 质溶液. 通过两个 $\mathrm{Ag} / \mathrm{AgCl}$ 电极在多孔膜两侧施加电 压, 利用皮安计(Keithley 6487)测量流过多孔膜的离子 电流. 电压扫描范围为从 $-2 \mathrm{~V}$ 到 $2 \mathrm{~V}$.

配置 $1 \mathrm{mmol} / \mathrm{L}$ 十二烷基硫醇(Aldrich)的乙醇溶液, 将纳米多孔金膜在该溶液中浸泡 $12 \mathrm{~h}$ 实现硫醇分子在 金表面的自组装修饰. 利用接触角仪(上海中晨)测量其 表面水接触角的大小. 利用皮安计测量多孔膜在 1 $\mathrm{mmol} / \mathrm{L} \mathrm{KF}$ 电解质中的电流-电压曲线. 在 $\mathrm{KF}$ 溶液中添 加不同浓度 $\left(10^{-3} 、 10^{-4} 、 10^{-5} 、 10^{-6} 、 10^{-7} 、 10^{-8}\right.$ 、 $10^{-9}$ 和 $\left.10^{-10} \mathrm{~mol} / \mathrm{L}\right)$ 的十二烷基苯磺酸钠 $(\mathrm{SDBS})$ 表面活 性剂, 测量多孔膜电流-电压曲线变化. 利用电流-电压 曲线中 $-2 \mathrm{~V}$ 电压下对应的离子电流值来计算纳米通道 在含有不同浓度表面活性剂 $\mathrm{KF}$ 水溶液中的电阻值 $\left(R_{\mathrm{m}}\right)$, 
从而描述疏水分子修饰的纳米多孔金膜对表面活性剂 的响应性.

\section{References}

[1] Hille, B. Ion Channels of Excitable Membranes, Sinauer Associates, Sunderland, 2001, pp. $1 \sim 21$.

[2] Tang, C.; Wang, L.; Yun, Y.; Zhang, C.; Liu, B. Acta Chim. Sinica 2011, 69, 343 (in Chinese). (唐橙橙, 王丽华, 贠延滨, 张陈淋, 刘 必前, 化学学报, 2011, 69, 343.)

[3] Lu, J.; Li, J. H. Angew. Chem. Int. Ed. 2015, 54, 13576.

[4] Cooper, G. M.; Hausman, R. E. The Cell: A Molecular Approach, Sinauer Associates, Sunderland, 2000, pp. $58 \sim 65$.

[5] Sigworth, F. J. Q. Rev. Biophys. 1994, 27, 1.

[6] Roux, B.; Berneche, S.; Egwolf, B.; Lev, B.; Noskov, Y.; Rowley, N.; Yu, H. J. Gen. Physiol. 2011, 137, 415.

[7] Hou, X.; Guo, W.; Jiang, L. Chem. Soc. Rev. 2011, 40, 2385.

[8] Hou, X.; Jiang, L. ACS Nano 2009, 3, 3339.

[9] Siwy, Z. S.; Howorka, S. Chem. Soc. Rev. 2010, 39, 1115.

[10] Xu, Y.; Meng, Z.; Zhai, J. Acta Chim. Sinica 2016, 74, 538 (in Chinese). (许阳蕾, 孟哲一, 翟锦, 化学学报, 2016, 74, 538.)

[11] Lan, W.; Holden, D. A.; White, H. S. J. Am. Chem. Soc. 2011, 133, 13300.

[12] White, H. S.; Bund, A. Langmuir 2008, 24, 2212.

[13] Liu, S.; Dong, Y.; Zhao, W.; Xie, X.; Ji, T.; Yin, X.; Liu, Y.; Liang, Z.; Momotenko, D.; Liang, D.; Girault, H.; Shao, Y. Anal. Chem. 2012, 84, 5565.

[14] Chen, P.; Mitsui, T.; Farmer, D. B.; Golovchenko, J.; Gordon, R. G.; Branton, D. Nano Lett. 2004, 4, 1333.

[15] Ali, M.; Yameen, B.; Cervara, J.; Ramírez, P.; Neumann, R.; Ensinger, W.; Knoll, W; Azzaroni, O. J. Am. Chem. Soc. 2010, 132, 8338 .

[16] Zhang, M.; Hou, X.; Wang, J.; Tian, Y.; Xia, F.; Zhai, J.; Jiang, L. Adv. Mater. 2012, 24, 2424.

[17] Ali, M.; Ramirez, P.; Nguyen, H. Q.; Nasir, S.; Cervera, J.; Mafe, S.; Ensinger, W. ACS Nano 2012, 6, 3631.

[18] Zhou, D.; Meng, Z.; Zhang, M.; Zhai, J. Acta Chim. Sinica 2015, 73, 716 (in Chinese). (周迪, 孟哲一, 张明辉, 翟锦, 化学学报, 2015, 73, 716.)

[19] Ali, M.; Nasir, S.; Ramirez, P.; Ahmed, I.; Nguyen, Q. H.; Fruk, L.; Mafe, S.; Ensinger, W. Adv. Funct. Mater. 2012, 22, 390.

[20] Guo, W.; Xia, H.; Xia, F.; Hou, X.; Cao, L.; Wang, L.; Xue, J.; Zhang, G.; Song, Y.; Zhu, D.; Wang, Y.; Jiang, L. Chem. Phys. Chem. 2010, 11, 859 .

[21] Vlassiouk, I.; Siwy, Z. Nano Lett. 2007, 7, 552.

[22] Xia, F.; Guo, W.; Hou, X.; Xue, J.; Xia, H.; Wang, L.; Song, Y.; Ji, H.; Ouyang, Q.; Wang, Y.; Jiang, L. J. Am. Chem. Soc. 2008, 130, 8345 .
[23] Jog, P. V.; Gin, M. S. Org. Lett. 2008, 10, 3693.

[24] Kumar, S. K.; Hong, J. D. Langmuir 2008, 24, 4190.

[25] Yameen, B.; Ali, M.; Neumann, R.; Ensinger, W.; Knoll, W. Azzaroni, O. Nano Lett. 2009, 9, 2788.

[26] Lin, L.; Liu, Y.; Yan, J.; Wang, X. S.; Li, J. H. Anal. Chem. 2013, 85, 334.

[27] Hou, X.; Guo, W.; Xia, F.; Nie, F.; Dong, H.; Tian, Y.; Wen, L.; Wang, L.; Cao, L.; Yang, Y.; Xue, J.; Song, Y.; Wang, Y.; Liu, D.; Jiang, L. J. Am. Chem. Soc. 2009, 131, 7800.

[28] Matsumoto, F.; Nishio, K.; Masuda, H. Adv. Mater. 2004, 16, 2105.

[29] Mara, A.; Siwy, Z.; Trautmann, C.; Wan, J.; Kamme, F. Nano Lett. 2004, 4, 497

[30] Hu, Z. Y.; Zhang, Q. Q.; Gao, J.; Liu, Z. Y.; Zhai, J.; Jiang, L. Langmuir 2013, 29, 4806.

[31] Haque, F.; Li, J.; Wu, H.; Liang, X.; Guo, P. Nano Today 2013, 8, 56

[32] Lin, L.; Yan, J.; Li, J. Anal. Chem. 2014, 86, 10546.

[33] Van Der Heyden, F. H.; Bonthuis, D. J.; Stein, D.; Meyer, C.; Dekker, C. Nano Lett. 2007, 7, 1022.

[34] Xie, Y.; Wang, X.; Xue, J.; Jin, K.; Chen, L.; Wang, Y. Appl. Phys. Lett. 2008, 93, 163116.

[35] Zhang, Q. Q.; Xiao, T. L.; Yan, N. N.; Liu, Z. Y.; Zhai, J.; Diao, X. G. Nano Energy 2016, 28, 188.

[36] Guo, W.; Cao, L.; Xia, J.; Nie, F.; Ma, W.; Xue, J.; Song, Y.; Zhu, D.; Wang, Y.; Jiang, L. Adv. Funct. Mater. 2010, 20, 1339.

[37] Wen, L.; Hou, X.; Tian, Y.; Zhai, J.; Jiang, L. Adv. Funct. Mater. 2010, 20, 2636

[38] Martin, R.; Nishizawa, M.; Jirage, K.; Kang, M.; Lee, S. Adv. Mater. 2001, 13, 1351.

[39] Vlassiouk, I.; Kozel, T. R.; Siwy, Z. S. J. Am. Chem. Soc. 2009, 131, 8211.

[40] Hou, X.; Dong, H.; Zhu, D.; Jiang, L. Small 2010, 3, 361.

[41] Siwy, Z. S.; Heins, E.; Harrell, C.; Kohli, P.; Martin, R. J. Am. Chem. Soc. 2004, 126, 10850.

[42] Lee, B.; Martin, R. J. Am. Chem. Soc. 2002, 124, 11850.

[43] Nishizawa, M.; Menon, P.; Martin, R. Science 1995, 268, 700.

[44] Yang, C.; Hinkle, P.; Menestrina, J.; Vlassiouk, I.; Siwy, Z. S. J. Phys. Lett. 2016, 7, 4152.

[45] Li, X. L.; Wang, Y.; Zhai, J. Acta Chim. Sinica 2016, 74, 597 (in Chinese). (李秀林, 汪洋, 翟锦, 化学学报, 2016, 74, 597.)

[46] Ding, Y.; Kim, Y.; Erlebacher, J. Adv. Mater. 2004, 16, 1897.

[47] Xu, C.; Su, J.; Xu, X.; Liu, P.; Zhao, H.; Tian, F.; Ding, Y. J. Am. Chem. Soc. 2007, 129, 42

[48] Erlebacher, J.; Aziz, M. J.; Karma, A.; Dimitrov, N.; Sieradzki, K. Nature 2001, 410, 450.

[49] Newman, R.; Sieradzki, K. Science 1994, 263, 1708.

[50] Polat, O.; Seker, E. J. Phys. Chem. C 2015, 119, 24812.

[51] Forty, A. J.; Durkin, P. Philos. Mag. A 1980, 42, 295.

[52] Zheng, D.; Hu, C.; Gan, T.; Dang, X.; Hu, S. Sens. Actuat. B-Chem. 2010, $148,247$. 